\title{
Critical Angles of the Nyström Method for Double Layer Potential Equation
}

\author{
Victor D. Didenko ${ }^{1}$ and Anh $\mathrm{My} \mathrm{Vu}^{2, *}$ \\ ${ }^{1}$ Faculty of Mathematics and Statisics, Ton Duc Thang University, Ho Chi Minh \\ City, Vietnam. \\ ${ }^{2}$ Faculty of Information Technology, Le Quy Don Technical University, 236 Hoang \\ Quoc Viet, Ha Noi, Vietnam.
}

Received 21 May 2017; Accepted (in revised version) 7 December 2017.

Abstract. The Nyström method for double layer potential equations on simple closed piecewise smooth contours is stable if and only if certain operators associated with the opening angles of the corners are invertible. Numerical experiments show that there are opening angles which cause the instability of the method.

AMS subject classifications: 65R20, 65N38, 45L05

Key words: Double layer potential equation, Nyström method, stability, critical angles.

\section{Introduction}

Boundary integral equations are widely used in approximate solution of partial differential equations - e.g. the Dirichlet problem for the Laplace equation

$$
\begin{aligned}
& \Delta u(x, y)=0, \quad(x, y) \in D \\
& u(x, y)=f(x, y), \quad(x, y) \in \Gamma
\end{aligned}
$$

in a simply connected domain $D \subset \mathbb{R}^{2}$ can be reduced to a double layer potential equation on the boundary $\Gamma$ of $D$.

We consider the double layer potential equation

$$
(A x)(t)=x(t)+\frac{1}{\pi} \int_{\Gamma} x(\tau) \frac{d}{d n_{\tau}} \log |t-\tau| d \Gamma_{\tau}+(T x)(t)=f(t), \quad t \in \Gamma .
$$

where $n_{\tau}$ refers to the outer normal to $\Gamma$ at the point $\tau \in \Gamma$ and $T$ is a compact operator. The form of $T$ depends on the boundary value problem studied. In particular, if the following integral representation

$$
u(z):=\frac{1}{\pi} \int_{\Gamma} x(\tau) \frac{d}{d n_{\tau}} \log |z-\tau| d \Gamma_{\tau}, \quad z \in D,
$$

${ }^{*}$ Corresponding author. Email addresses: viktor.didenko@tdt.edu.vn (V.D. Didenko), anhmy7284@ gmail. com (A.M. Vu) 
of the solutions of (1.1) is used, then $T$ is the zero operator [2]. The operator $A$ in (1.2) has been thoroughly studied for various contours and spaces - cf. Refs. [8,22, 25, 27]. For smooth curves $\Gamma$, the double layer potential operator

$$
V_{\Gamma} x(t):=\frac{1}{\pi} \int_{\Gamma} x(\tau) \frac{d}{d n_{\tau}} \log |t-\tau| d \Gamma_{\tau}
$$

acting in an $L^{p}$-space is compact. This property of $V_{\Gamma}$ substantially simplifies the stability analysis of approximation methods. In fact, for smooth curves, the invertibility of $A$ and a good convergence of approximation operators to $A$ ensure the stability of the method. For curves with corners, the operator (1.3) is not compact and stability depends on additional parameters - cf. Refs. $[2,12,13,18,21,22]$. Here we consider the Nyström discretisation based on Gauss-Legendre quadrature formula. This method is easily implemented and demonstrates a good convergence even for contours with a large set of corners cf. Refs. $[4,5,20]$. Its stability for the Sherman-Lauricella and Muskhelishvili equations has been studied earlier $[9-11,15]$. For the double layer potential equation, the stability is proven for sufficiently nice curves - polygons or curves coinciding with polygons in neighbourhoods of corners, or/and for sufficiently nice double layer potential operators perturbations of the identity by compact and small norm operators [18,21,22]. Besides, so far only sufficient stability conditions are available. Our goal here is to establish necessary and sufficient stability conditions of this method for general simple piecewise smooth curves and to provide a procedure for their verification. It turns out that there exist angles which cause instability. Such angles are called critical and if $\Gamma$ has a critical angle, the Nyström method is not stable regardless of the shape of the curve. In contrast, spline Galerkin methods do not have any critical angles $[15,17]$ but the complexity of the implementation and computational cost are significantly higher than for Nyström methods. Therefore, in practical computations Nyström type methods are preferable and there are modifications designed to handle the instability induced by non-smooth boundaries $[7,19]$. On the other hand, in the interval $[0.1 \pi, 1.9 \pi]$, the Nyström method for the double layer potential equation has only four critical angles. It is possible that some or all of them are irrational, so we may never encounter the unstable situation during implementation of the method cf. Remark 3.1 and Fig. 3 below.

Let us make a few technical remarks. We identify every point $(x, y)$ of $\mathbb{R}^{2}$ with the point $z=x+i y$ in the complex plane $\mathbb{C}$. By $S_{\Gamma}$ we denote the Cauchy singular integral operator on $\Gamma$,

$$
\left(S_{\Gamma} x\right)(t):=\frac{1}{\pi i} \int_{\Gamma} \frac{x(\tau) d \tau}{\tau-t}
$$

and by $M$ the operator of complex conjugation, $M \varphi(t):=\overline{\varphi(t)}$. It is known [23] that the double layer potential operator $V_{\Gamma}$ can be represented in the form

$$
V_{\Gamma}=\frac{1}{2}\left(S_{\Gamma}+M S_{\Gamma} M\right)
$$


so that the equation (1.2) becomes

$$
A x=\left(I+\frac{1}{2} S_{\Gamma}+\frac{1}{2} M S_{\Gamma} M+T\right) x=f .
$$

We consider this equation in the space $L^{2}:=L^{2}(\Gamma)$ of all complex-valued Lebesgue measurable functions $f$ with the norm

$$
\|f\|_{L^{2}}:=\left(\int_{\Gamma}|f(t)|^{2}|d t|\right)^{1 / 2} .
$$

The Nyström method we study, is based on the composite Gauss-Legendre quadrature rule

$$
\int_{0}^{1} u(s) d s \approx \sum_{l=0}^{n-1} \sum_{p=0}^{d-1} w_{p} u\left(s_{l p}\right) / n
$$

where

$$
s_{l p}=\frac{l+\varepsilon_{p}}{n}, \quad l=0,1, \cdots, n-1, p=0,1, \cdots, d-1,
$$

and $w_{p}$ and $0<\varepsilon_{0}<\varepsilon_{1}<\cdots<\varepsilon_{d-1}<1$ are, respectively, Gauss-Legendre weights and Gauss-Legendre points rescaled to the interval $[0,1]$. A more detailed description of the approximation method is given in Section 2. The stability of the method is studied in Section 3. We prove that the corresponding Nyström method is stable if and only if some operators $B_{\omega}, \omega \in(0,2 \pi)$ depending on the corners are invertible. These operators belong to a Toeplitz algebra and in general no effective conditions of their invertibility are available. Nevertheless, for $\omega \in[0.1 \pi, 1.9 \pi]$ a numerical approach introduced in Section 4, allows us to find all irreversible operators $B_{\omega}$, hence locate the critical angles. Small opening angles and angles close to $2 \pi$ can also be scrutinised but it requires more efforts and computational cost is high.

\section{Nyström Method}

Let $\Gamma$ be a simple piecewise smooth positively oriented contour in the complex plane $\mathbb{C}$ and let $\gamma: \mathbb{R} \mapsto \mathbb{C}$ be a 1-periodic parametrization of $\Gamma$. By $\mathfrak{M}_{\Gamma}$ we denote the set of all corner points $\tau_{0}, \tau_{1}, \cdots, \tau_{q-1}$ of $\Gamma$. Assume that

$$
\tau_{j}=\gamma(j / q), \quad j=0,1, \cdots, q-1,
$$

the function $\gamma$ is two times continuously differentiable on each subinterval $(j / q,(j+1) / q)$ and

$$
\left|\gamma^{\prime}(j / q+0)\right|=\left|\gamma^{\prime}(j / q-0)\right|, \quad j=0,1, \cdots, q-1 .
$$

Choose an $n=r q, r \in \mathbb{N}$ and a non-negative integer $d$ such that $n>d+1$. By $S_{n}^{d}(\Gamma)$ we denote the space of the smoothest splines of degree $d$ on $\Gamma$ associated with the parametrization $\gamma$. More precisely, let $\varphi^{d}=\varphi^{d}(s), s \in \mathbb{R}$ be the uniform cardinal B-spline of degree $d$. 
Recall [26] that $\varphi^{0}=\chi_{[0,1)}$, where $\chi_{[0,1)}$ is the characteristic function of the interval $[0,1)$ and for $d \geq 1$, the functions $\varphi^{d}$ can be obtained by the recursive formula

$$
\varphi^{d}(s)=\int_{\mathbb{R}} \chi_{[0,1)}(s-x) \varphi^{d-1}(x) d x .
$$

For any fixed non-negative integer $d$ and for any $n=m d$, we define the functions

$$
\psi_{k n}(s):=\psi(n s-k), \quad k \in \mathbb{Z}, \quad s \in \mathbb{R},
$$

where $\psi(s):=\varphi^{d}(s)$, and consider the set

$$
\Lambda^{d}:=\left\{\psi_{k n}: k \geq 0 \text { and } \operatorname{supp}\left\{\psi_{k n}\right\} \subset(0,1)\right\} .
$$

It follows from Lemma 5.4.1 in Ref. [14] that for $d \geq 1$ the support $\operatorname{supp}\left\{\psi_{k n}\right\}$ of the function $\psi_{k n}$ is the interval $(k / n,(k+d+1) / n)$ hence $\psi_{k n} \in \Lambda^{d}$ if and only if $0 \leq k \leq$ $n-d-1$. If $n$ is sufficiently large, then the restriction $\left.\psi_{k n}\right|_{[0,(1+d) / n)}$ of $\psi_{k n}$ to $[0,(1+d) / n)$ can be extended to the whole real line $\mathbb{R}$ in a 1-periodic way. Such an extension is denoted by the same symbol $\psi_{k n}$. Now using a 1-periodic parametrization $\gamma=\gamma(s), s \in \mathbb{R}$ of the contour $\Gamma$, we set

$$
\widetilde{\psi}_{k n}(t):=\psi_{k n}(s), \quad t=\gamma(s) \in \Gamma .
$$

If $(d+1) / n<1$, this definition is correct, and by $S_{n}^{d}=S_{n}^{d}(\Gamma)$ we denote the corresponding spline space on $\Gamma-$ i.e. $S_{n}^{d}(\Gamma) \subset L^{2}(\Gamma)$ is the set of all linear combinations of the functions $\widetilde{\psi}_{k n}, k=0,1, \cdots, n-1$.

We assume that $n=m d$ and $m=q r, m, r \in \mathbb{N}$ and consider the set

$$
\tau_{l p}=\gamma\left(\frac{l+\varepsilon_{p}}{n}\right), \quad l=0,1, \cdots, n-1, \quad p=0,1, \cdots, d-1,
$$

where $0<\varepsilon_{0}<\varepsilon_{1}<\cdots<\varepsilon_{d-1}<1$ are the Gauss-Legendre points rescaled to the interval $[0,1]$. If the integral operator

$$
K \varphi(t):=\int_{\Gamma} k(t, \tau) \varphi(\tau) d \tau
$$

has a sufficiently smooth kernel $k$ and if $\varphi$ is a Riemann integrable function, then this operator can be approximated by the quadrature rule (1.5) — viz.

$$
\begin{aligned}
& \int_{\Gamma} k(t, \tau) \varphi(\tau) d \tau=\int_{0}^{1} k(\gamma(\sigma), \gamma(s)) \varphi(\gamma(s)) \gamma^{\prime}(s) d s \\
& \approx K^{(\varepsilon, n)} \varphi(t)=\sum_{l=0}^{n-1} \sum_{p=0}^{d-1} w_{p} k\left(t, \tau_{l p}\right) \varphi\left(\tau_{l p}\right) \tau_{l p}^{\prime} / n,
\end{aligned}
$$

with $\tau_{l p}^{\prime}:=\gamma^{\prime}\left(\left(l+\varepsilon_{p}\right) / n\right)$. 
Let $\mathbf{R}(\Gamma)$ stand for the set of all Riemann integrable functions on $\Gamma$ and $Q_{n}^{\varepsilon}: L_{\infty}(\Gamma) \mapsto$ $S_{n}^{d}(\Gamma)$ be the interpolation projection on the space $S_{n}^{d}(\Gamma)$ such that

$$
Q_{n}^{\varepsilon} x\left(\tau_{l p}\right):=x\left(\tau_{l p}\right), \quad l=0,1, \cdots, n-1, p=0,1, \cdots, d-1,
$$

for all $x \in \mathbf{R}(\Gamma)$. If $\varepsilon_{p} \neq 0.5, p=0,1, \cdots, d-1$, then such projection operator $Q_{n}^{\varepsilon}$ exists and the sequence $\left(Q_{n}^{\varepsilon}\right)_{n \in \mathbb{N}}: \mathbf{R}(\Gamma) \mapsto L^{2}(\Gamma)$ converges strongly to the corresponding embedding operator [24, Chapter 2] - viz.

$$
\lim _{n \rightarrow \infty}\left\|Q_{n}^{\varepsilon} f-f\right\|_{L^{2}(\Gamma)}=0, \quad f \in \mathbf{R}(\Gamma)
$$

We also consider the orthogonal projections $P_{n}: L^{2}(\Gamma) \rightarrow S_{n}^{d}(\Gamma), n \in \mathbb{N}$ and note that the sequence $\left(P_{n}\right)$ converges strongly to the identity operator - cf. Ref. [24].

It is convenient to drop the compact operator in (1.2) and study the stability of the Nyström method for the equation

$$
A_{\Gamma} x=\left(I+V_{\Gamma}\right) x=f .
$$

This simplifies notation but does not influence the proof of the main result. Approximate solutions $x_{n}, n \in \mathbb{N}$ of (2.6) are derived from the operator equations

$$
Q_{n}^{\varepsilon} A_{\Gamma}^{(\varepsilon, n)} P_{n} x_{n}:=Q_{n}^{\varepsilon} P_{n} x_{n}+Q_{n}^{\varepsilon} V_{\Gamma}^{(\varepsilon, n)} P_{n} x_{n}=Q_{n}^{\varepsilon} f, \quad x_{n} \in S_{n}^{d}(\Gamma), n \in \mathbb{N},
$$

which are equivalent to the following systems of linear algebraic equations:

$$
\begin{aligned}
& x\left(\tau_{k r}\right)+\frac{1}{2 \pi i} \sum_{l=0}^{n-1} \sum_{p=0}^{d-1} w_{p} x\left(\tau_{l p}\right)\left(\frac{\tau_{l p}^{\prime}}{\tau_{l p}-\tau_{k r}}-\frac{\overline{\tau_{l p}^{\prime}}}{\overline{\tau_{l p}}-\overline{\tau_{k r}}}\right) \frac{1}{n}=f\left(\tau_{k r}\right) \\
& k=0,1, \cdots, n-1, r=0,1, \cdots, d-1 .
\end{aligned}
$$

Remark 2.1. Let $\mathrm{k}=\mathrm{k}(t, \tau)$ denote the kernel of the double layer potential operator $V_{\Gamma}$. Straightforward calculations [16] show that for any $\tau \notin \mathfrak{M}_{\Gamma}$,

$$
\lim _{t \rightarrow \tau} \mathrm{k}(t, \tau)=\frac{\iota \operatorname{Im}\left(\gamma^{\prime}(s) \overline{\gamma^{\prime \prime}(s)}\right)}{\left|\gamma^{\prime}(s)\right|^{2}}, \quad \tau=\gamma(s) .
$$

Therefore, if $l=k$ and $p=r$, then the expression in the brackets of (2.7) is set to be $\gamma^{\prime}\left(s_{l p}\right) \lim _{t \rightarrow \tau_{l p}} \mathrm{k}\left(t, \tau_{l p}\right)$.

Let us find approximate solutions of the equation (2.6) with continuous and discontinuous right-hand sides for two different contours $\mathscr{L}_{1}=\mathscr{L}_{1}(\omega) \mathscr{L}_{2}=\mathscr{L}_{2}(\omega), \omega \in(0,2 \pi)$, the graphs of which are given in Fig. 1 . The curves $\mathscr{L}_{1}$ and $\mathscr{L}_{2}$ have, respectively, one and two corners of the same magnitude $\omega \in(0,2 \pi)$ and are defined by

$$
\mathscr{L}_{j}:=\left\{t \in \mathbb{C}: t=\gamma_{j}(s), \quad s \in[0,1]\right\}, \quad j=1,2,
$$



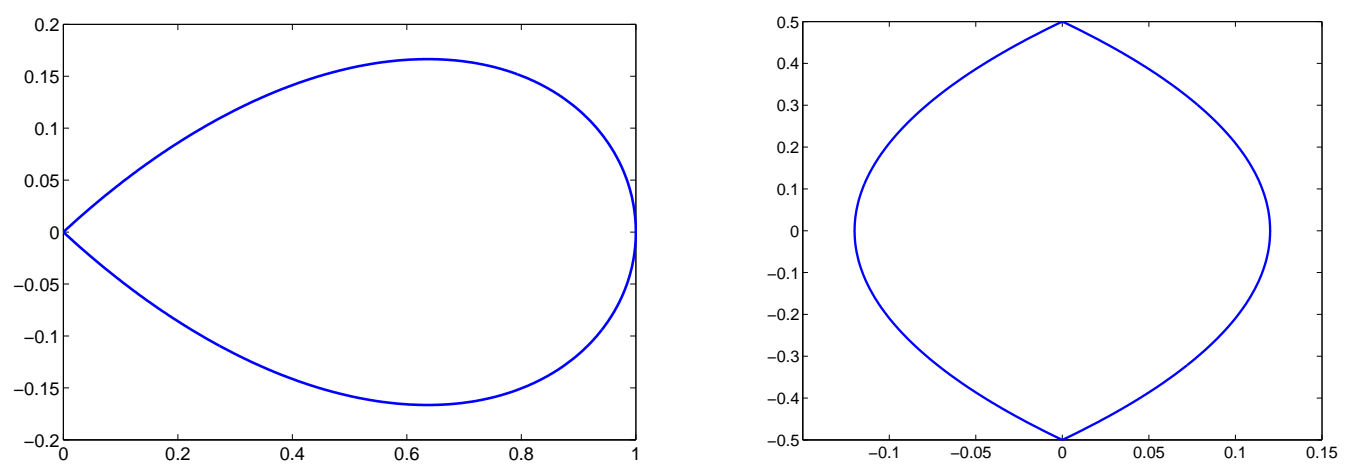

Figure 1: Left: one-corner curve $\mathscr{L}_{1}$. Right: two-corner curve $\mathscr{L}_{2}$.

Table 1: Convergence of the Nyström method.

\begin{tabular}{|c|c|c||c|c|}
\hline$n$ & $E_{n}^{\left(f_{1}, \mathscr{L}_{1}\right)}$ & $E_{n}^{\left(f_{1}, \mathscr{L}_{2}\right)}$ & $E_{n}^{\left(f_{2}, \mathscr{L}_{1}\right)}$ & $E_{n}^{\left(f_{2}, \mathscr{L}_{2}\right)}$ \\
\hline 32 & $2.5 \times 10^{-3}$ & $2.6 \times 10^{-3}$ & $1.5 \times 10^{-2}$ & $2.0 \times 10^{-2}$ \\
\hline 96 & $8.3 \times 10^{-4}$ & $1.1 \times 10^{-3}$ & $7.5 \times 10^{-3}$ & $1.3 \times 10^{-2}$ \\
\hline 256 & $3.1 \times 10^{-4}$ & $2.1 \times 10^{-4}$ & $4.0 \times 10^{-3}$ & $7.3 \times 10^{-3}$ \\
\hline
\end{tabular}

where

$$
\begin{aligned}
& \gamma_{1}(s)=\sin (\pi s) \exp (i \omega(s-0.5)), \quad s \in[0,1], \\
& \gamma_{2}(s)= \begin{cases}-\frac{1}{2} \cot (\omega / 2)+\frac{1}{2 \sin (\omega / 2)} \exp (i \omega(2 s-0.5)) & \text { if } 0 \leq s \leq 1 / 2 \\
\frac{1}{2} \cot (\omega / 2)-\frac{1}{2 \sin (\omega / 2)} \exp (i \omega(2 s-1.5)) & \text { if } 1 / 2<s \leq 1\end{cases}
\end{aligned}
$$

Further, let $f_{1}$ and $f_{2}$ be the following functions

$$
f_{1}(z)=-z|z|
$$

and

$$
f_{2}(z)=\left\{\begin{array}{rr}
-1+i z & \text { if } \operatorname{Im} z<0 \\
1+i z & \text { if } \operatorname{Im} z \geq 0
\end{array}\right.
$$

The function $f_{1}$ is continuous everywhere, while $f_{2}$ has discontinuities one of which is located at the angular point of $\mathscr{L}_{1}$.

The double layer potential equation with the right-hand sides $f_{1}$ and $f_{2}$ is solved by the Nyström method (2.7) with $d=16$, and approximate solutions are shown in Fig. 2. Table 1 demonstrates the convergence of the method. We note that

$$
E_{n}^{\left(f_{k}, \mathscr{L}_{j}\right)}:=\frac{\left\|x_{2 n}-x_{n}\right\|_{2}}{\left\|x_{2 n}\right\|_{2}},
$$



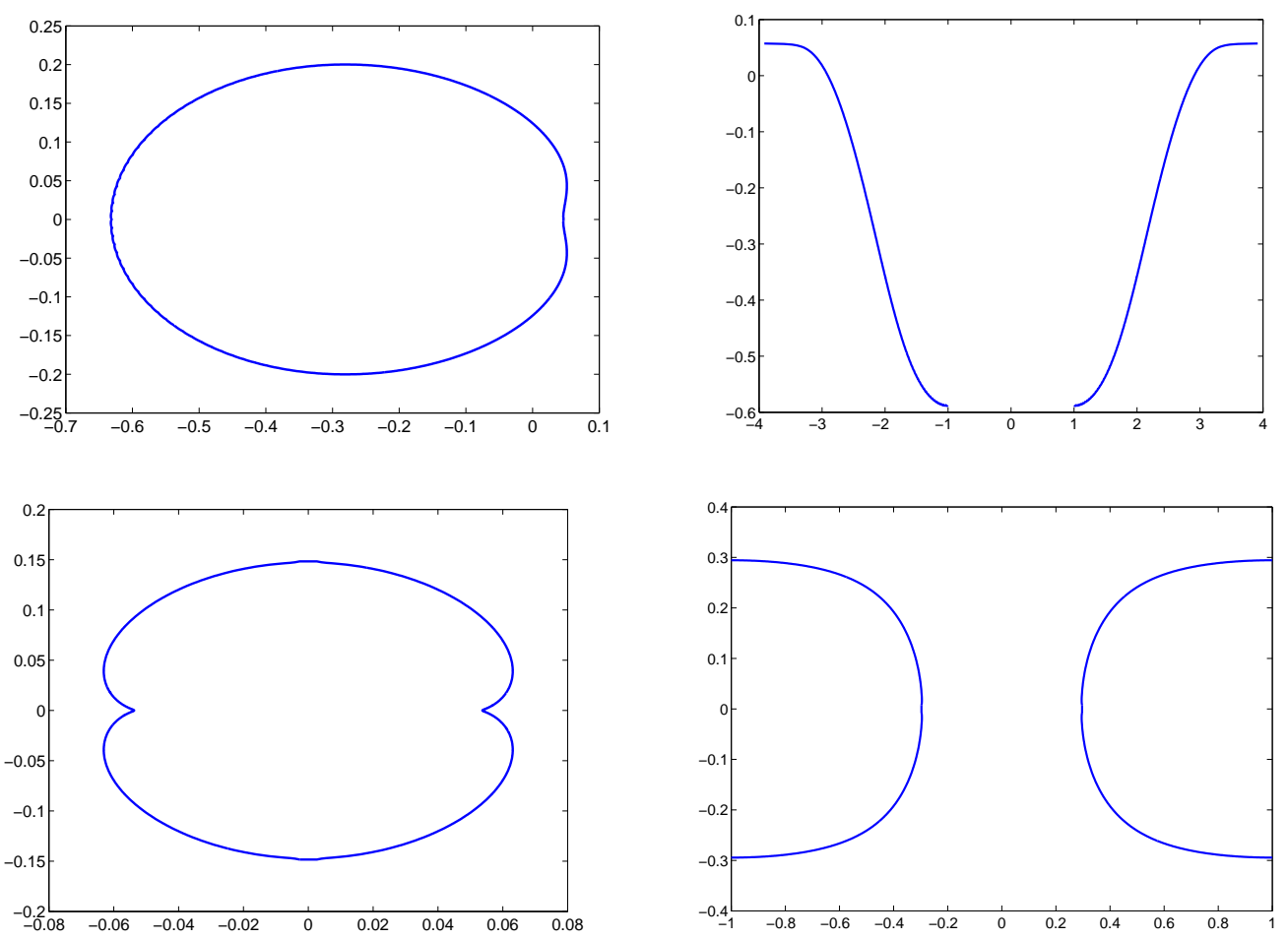

Figure 2: Approximate solutions of equation (2.6) obtained by Nyström method (2.7) (Real part versus imaginary part), $n=512, d=16$. First row: Solutions of (2.6) on $\mathscr{L}_{1}$. Second row: Solutions of (2.6) on $\mathscr{L}_{2}$. Left: Solutions for continuous r.-h.s. $f_{1}(z)$. Right: Solutions for discontinuous r.-h.s. $f_{2}(z)$.

where $x_{n}$ is the approximate solution of the equation (2.6) for the contour $\mathscr{L}_{j}(0.3 \pi), j=$ 1,2 with the right-hand side $f_{k}, k=1,2$.

The convergence can be improved by using modifications of the Nyström method [3$5,20]$ but the focus of this paper is on the stability and critical angles.

\section{Stability}

It is well-known that the invertibility of $A$ is a necessary condition for applicability of many numerical methods $[19,24]$. It depends on the contour $\Gamma$, compact operator $T$ and the space where $A$ acts. In this section we describe a more general kind of invertibility viz. the Fredholmness of the operator $A$. These results are not new but during the proof we also introduce various operators needed in the analysis of Nyström method.

Let $\gamma$ be a parametrization of a piecewise smooth curve $\Gamma$ satisfying the relations (2.1)(2.2). By $\omega_{j}$ we denote the angle between two semi-tangents to $\Gamma$ at the corner $\tau_{j}$ and by $\beta_{j}$ the angle between the right semi-tangent and the real axis $\mathbb{R}$. We also consider contour

$$
\Gamma_{j}:=\mathbb{R}^{-} e^{i\left(\beta_{j}+\omega_{j}\right)} \bigcup \mathbb{R}^{+} e^{i \beta_{j}},
$$


where $\mathbb{R}^{-}$and $\mathbb{R}^{+}$are positive semi-axes directed to and away from the origin, respectively. By $L^{2}\left(\Gamma_{j}\right)$ we denote $L^{2}$-space on $\Gamma_{j}$, and with each corner point $\tau_{j} \in \Gamma$ we associate the operator $A_{\Gamma_{j}}:=I+V_{\Gamma_{j}}: L^{2}\left(\Gamma_{j}\right) \rightarrow L^{2}\left(\Gamma_{j}\right)$, where $V_{\Gamma_{j}}$ is the double layer potential operator on $\Gamma_{j}$.

The application of Theorem 1.9.5 in [14] to the operator (1.4) leads to the following result.

Proposition 3.1. Let $\Gamma$ be a simple closed piecewise smooth curve in the complex plane $\mathbb{C}$. The operator $A$ is Fredholm if and only if the operators $A_{\Gamma_{j}}: L^{2}\left(\Gamma_{j}\right) \rightarrow L^{2}\left(\Gamma_{j}\right)$ are invertible for all $j=0,1, \cdots, q-1$.

Let $\mathbb{M}$ and $\mathbb{M}^{-1}$ denote, respectively, direct and inverse Mellin transforms — i.e.

$$
(\mathbb{M} f)(z)=\int_{0}^{+\infty} x^{1 / p+\alpha-z i-1} f(x) d x, \quad\left(\mathbb{M}^{-1} f\right)(x)=\frac{1}{2 \pi} \int_{-\infty}^{+\infty} x^{z i-1 / p-\alpha} f(z) d z .
$$

The Mellin convolution operator $\mathscr{M}(b)$ with the symbol $b$ is defined by

$$
\mathscr{M}(b) x(\sigma)=\left(\left(\mathbb{M}^{-1} b \mathbb{M}\right) x\right)(\sigma)
$$

and for some classes of symbols $b$, this operator can be represented in the integral form

$$
\mathscr{M}(b) x(\sigma)=\int_{0}^{+\infty} \mathbf{k}\left(\frac{\sigma}{s}\right) x(s) \frac{d s}{s},
$$

with the kernel $\mathbf{k}=\mathbb{M}^{-1} b$.

Let $\mathscr{N}_{\omega}: L^{2}\left(\mathbb{R}^{+}\right) \rightarrow L^{2}\left(\mathbb{R}^{+}\right)$be the operator defined by

$$
\left(\mathscr{N}_{\omega}(\phi)\right)(\sigma)=\frac{1}{\pi i} \int_{0}^{+\infty} \frac{\phi(s) d s}{s-\sigma e^{i \omega}} .
$$

It is easily seen that $A_{\Gamma_{j}}$ is isometrically isomorphic to the matrix operator

$$
A_{\omega_{j}}=\left(\begin{array}{cc}
I & (1 / 2)\left(\mathscr{N}_{\omega}-\mathscr{N}_{2 \pi-\omega}\right) \\
(1 / 2)\left(\mathscr{N}_{\omega}-\mathscr{N}_{2 \pi-\omega}\right) & I
\end{array}\right)
$$

acting on the space $L^{2}\left(\mathbb{R}^{+}\right)^{2}:=L^{2}\left(\mathbb{R}^{+}\right) \times L^{2}\left(\mathbb{R}^{+}\right)$, and the corresponding isomorphism is $A \mapsto \eta A \eta^{-1}$ with the mapping $\eta: L^{2}\left(\Gamma_{j}\right) \mapsto L^{2}\left(\mathbb{R}^{+}\right)^{2}$ defined by

$$
\eta(f)(s)=\left(f\left(s e^{i\left(\beta_{j}+\omega_{j}\right)}\right), f\left(s e^{i \beta_{j}}\right)\right)^{T}, s \in \mathbb{R}^{+} .
$$

It is well-known $[14,19,25]$ that $\mathscr{N}_{\omega}$ is the Mellin convolution operator $\mathscr{M}\left(\mathbf{n}_{\omega}\right)$ with the symbol

$$
\mathbf{n}_{\omega_{j}}(y)=\frac{e^{\left(\pi-\omega_{j}\right) y}}{\sinh \pi y}, \quad y=z+\frac{i}{2}, z \in \mathbb{R}
$$


which leads to the formula

$$
\operatorname{smb}\left((1 / 2)\left(\mathscr{N}_{\omega_{j}}-\mathscr{N}_{2 \pi-\omega_{j}}\right)\right)=\frac{\sinh \left(\pi-\omega_{j}\right) y}{\sinh \pi y}, \quad y=z+\frac{i}{2}, \quad z \in \mathbb{R}
$$

so that

$$
\operatorname{smb} A_{\omega_{j}}(y)=\left(\begin{array}{cc}
1 & \frac{\sinh \left(\pi-\omega_{j}\right) y}{\sinh \pi y} \\
\frac{\sinh \left(\pi-\omega_{j}\right) y}{\sinh \pi y} & 1
\end{array}\right) .
$$

The Mellin operator $(1 / 2)\left(\mathscr{N}_{\omega_{j}}-\mathscr{N}_{2 \pi-\omega_{j}}\right)$ can be also represented in the integral form (3.1) with the kernel

$$
\mathbf{k}(z)=\mathbf{k}_{\omega_{j}}(z)=\frac{1}{\pi i} \frac{i z \sin \omega_{j}}{\left(1-z e^{i \omega_{j}}\right)\left(1-z e^{-i \omega_{j}}\right)}
$$

Corollary 3.1. If $\Gamma$ is a simple closed piecewise smooth contour satisfying the conditions of Section 2, then the operator $A: L^{2}(\Gamma) \rightarrow L^{2}(\Gamma)$ of (1.4) is Fredholm.

Proof. The matrix Mellin operator $A_{\omega_{j}}$ is invertible in $L^{2}(\Gamma)$ if and only if its symbol (3.4) is invertible. The determinant of $\operatorname{smb} A_{\omega_{j}}$ is $1-\sinh ^{2}\left(\pi-\omega_{j}\right) y / \sinh ^{2} \pi y$ and is equal to zero if and only if $\sinh \left(\pi-\omega_{j}\right) y=\sinh \pi y \operatorname{or} \sinh \left(\pi-\omega_{j}\right) y=-\sinh \pi y$. Let us consider the first of these equations. Separation of the real and imaginary parts produces the equations

$$
\begin{aligned}
& \cosh \left(\left(\pi-\omega_{j}\right) z\right) \sin \frac{\pi-\omega_{j}}{2}=\cosh (\pi z) \\
& \sinh \left(\left(\pi-\omega_{j}\right) z\right) \cos \frac{\pi-\omega_{j}}{2}=0,
\end{aligned}
$$

where $z \in \mathbb{R}$. Since $\cos \left(\left(\pi-\omega_{j}\right) / 2\right) \neq 0$ for any $\omega_{j} \in(0,2 \pi)$, the second equation is satisfied only if $z=0$ or $\pi-\omega_{j}=0$. For $z=0$, the first equation takes the form $\sin ((\pi-$ $\left.\left.\omega_{j}\right) / 2\right)=1$ and has no solution in the interval $(0,2 \pi)$. On the other hand, if $\pi-\omega_{j}=0$, the first equation transforms into $\cosh (\pi z)=0$ and is also not solvable. Thus the symbol of $A_{\omega_{j}}$ does not vanish on the line $\mathbb{R}+i / 2$, hence $A_{\omega_{j}}$ are invertible for all $j=0,1, \cdots, q-1$ and so are the operators $A_{\Gamma_{j}}$. The application of Proposition 3.1 finishes the proof.

We proceed with the stability of the method (2.7). Let $\left(A_{n}\right)$ be a bounded sequence of linear bounded operators $A_{n}: S_{n}^{d}(\Gamma) \mapsto S_{n}^{d}(\Gamma)$. The set $\mathscr{T}$ of such sequences equipped with componentwise operations of addition, multiplication, involution and multiplication by scalars, and with the norm

$$
\left\|\left(A_{n}\right)\right\|:=\sup _{n \in \mathbb{N}}\left\|A_{n}\right\|
$$

becomes a $C^{*}$-algebra.

Definition 3.1. The sequence $\left(A_{n}\right) \in \mathscr{T}$ is called stable if there is an $n_{0} \in \mathbb{N}$ such that for all $n \geq n_{0}$ the operators $A_{n} P_{n}: S_{n}^{d}(\Gamma) \mapsto S_{n}^{d}(\Gamma)$ are invertible and the norms $\left\|\left(A_{n} P_{n}\right)^{-1} P_{n}\right\|_{n \geq n_{0}}$ are uniformly bounded. 


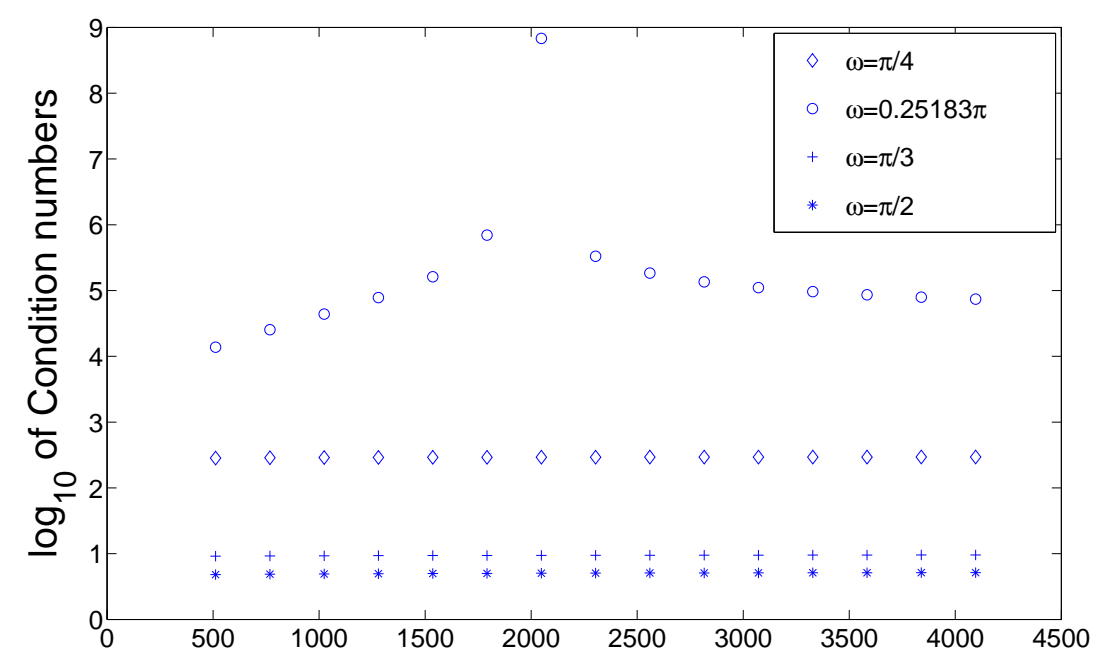

Figure 3: The Nyström method: condition numbers versus the number of discretisation points for contour $\mathscr{L}_{1}(\omega)$ with $\omega=0.25183 \pi, \pi / 3, \pi / 4, \pi / 2$.

Remark 3.1. The stability of approximation methods is directly connected to the condition numbers of the corresponding matrix sequences. Fig. 3 shows the condition numbers for the matrices representing the Nyström method for the double layer potential operator on the contour $\mathscr{L}_{1}(\omega)$ with $\omega=0.25183 \pi, \pi / 3, \pi / 4, \pi / 2$. An abnormality of the graph in the case $\omega=0.25183 \pi$ is caused by the proximity of this point to a critical angle. Nevertheless, since $0.25183 \pi$ is only an approximation of that angle, the sequence of condition numbers stabilises as $\mathrm{n}$ grows.

It is well known that the stability of the approximation method $\left(A_{n}\right)$ is equivalent to the invertibility of the coset $\left(A_{n}\right)+\mathscr{G}$ in the quotient algebra $\mathscr{T} / \mathscr{G}$ - cf. Refs. [14, 19, 24, 25], where $\mathscr{G}$ is the set of all bounded sequences uniformly convergent to zero,

$$
\mathscr{G}=\left\{\left(G_{n}\right) \in \mathscr{T}: \lim _{n \rightarrow \infty}\left\|G_{n}\right\|=0\right\} .
$$

In many cases, quotient algebra $\mathscr{T} / \mathscr{G}$ is too large to treat the invertibility problem efficiently. Therefore, one often considers a smaller algebra $\mathscr{A} \subset \mathscr{T}$ of sequences containing the approximation method studied, simultaneously expanding $\mathscr{G}$ to a larger ideal $\mathscr{J}$ in such a way that the initial problem is equivalent to the invertibility of the corresponding coset in the quotient algebra $\mathscr{A} / \mathscr{J}$. More precisely, let $\mathscr{B}\left(L^{2}(\Gamma)\right)$ be the set of all linear bounded operators acting in the space $L^{2}(\Gamma)$. By $\mathscr{A} \subset \mathscr{T}$ we denote the close subalgebra of $\mathscr{T}$ containing all sequences $\left(A_{n}\right)$ such that there is an operator $A \in \mathscr{B}\left(L^{2}(\Gamma)\right)$ for which the strong limits

$$
s-\lim _{n \rightarrow \infty} A_{n} P_{n}=A, \quad s-\lim _{n \rightarrow \infty}\left(A_{n}\right)^{*} P_{n}=A^{*}
$$

exist. Moreover, if $\mathscr{K}\left(L^{2}(\Gamma)\right)$ is the set of all compact operators on $L^{2}(\Gamma)$, then the family 
of sequences

$$
\mathscr{J}=\left\{\left(J_{n}\right): J_{n}=P_{n} K P_{n}+G_{n}, K \in \mathscr{K}\left(L^{2}(\Gamma)\right), G_{n} \in \mathscr{G}\right\} .
$$

is a closed two-sided ideal of $\mathscr{A}$.

Theorem 3.1 (cf. Refs. $[14,24,25])$. A sequence $\left(A_{n}\right) \in \mathscr{A}$ is stable if and only if the operator $A$ is invertible in $\mathscr{B}\left(L^{2}(\Gamma)\right)$ and the coset $\left(A_{n}\right)+\mathscr{J}$ is invertible in the quotient algebra $\mathscr{A} / \mathscr{J}$.

We use this result to study the applicability of the Nyström method to the double layer potential equations. Note that the sequence of approximation operators $\left(A_{n}\right)_{n \in \mathbb{N}}$ corresponding to the Nyström method strongly converges to the operator $A_{\Gamma}-$ cf. Ref. [24, Chapter 2] and Ref. [19, Chapter 5]. Similar statement is valid for the sequence of adjoint operators. To show the invertibility of the coset $\left(A_{n}\right)+\mathscr{J}$ in the quotient algebra $\mathscr{T} / \mathscr{J}$ we use a local principle. With each point $\tau \in \Gamma$ we associate a simpler sequence of approximation operators $\left(A_{n}^{\tau}\right)$, an algebra $\mathscr{A}_{\tau}$ and ideal $\mathscr{J}_{\tau}$ such that the invertibility of the coset $\left(A_{n}\right)+\mathscr{J}$ in $\mathscr{T} / \mathscr{J}$ is equivalent to the invertibility of the coset $\left(A_{n}\right)+\mathscr{J}_{\tau}$ in $\mathscr{A}_{\tau} / \mathscr{J}_{\tau}$ for all $\tau \in \Gamma$. For more detail we refer the reader to Refs. $[14,19,24,25]$. If $\tau \notin \mathfrak{M}_{\Gamma}$ and $U_{\tau} \subset \Gamma$ is a neighbourhood of $\tau$ such that $\mathfrak{M}_{\Gamma} \cap U_{\tau}=\emptyset$ and if $f_{\tau}$ is a function continuous on $\Gamma$ such that

$$
f_{\tau}(t)= \begin{cases}1 & \text { if } t=\tau \\ 0 & \text { if } t \in \Gamma \backslash U_{\tau},\end{cases}
$$

then the operator $f_{\tau} V_{\Gamma} f_{\tau} \in \mathscr{K}\left(L^{2}(\Gamma)\right)-$ cf. Corollary 4.6.3 in [25]. Therefore, the sequence $\left(A_{n}^{\tau}\right)$ is locally equivalent to the sequence generated by the projections $\left(P_{n}\right)$, so that the corresponding coset containing the sequence $\left(A_{n}^{\tau}\right)$ is invertible. Thus one only has to identify and study the cosets associated with the corners of $\Gamma$. To this end, for each corner points $\tau_{j} \in \Gamma$ we consider the corresponding approximation method for the operator $A_{\omega_{j}}$ of (3.2), approximating the integrals $\int_{\Gamma_{j}} x(\tau) d \tau$ similarly to (1.5) - i.e.

$$
\begin{aligned}
\int_{\Gamma_{j}} x(\tau) d \tau \approx & \sum_{l=-\infty}^{-1} \sum_{p=0}^{d-1} w_{p} x\left(\frac{l+\varepsilon_{p}}{n} e^{i\left(\beta_{j}+\omega_{j}\right)}\right) \frac{e^{i\left(\beta_{j}+\omega_{j}\right)}}{n} \\
& +\sum_{l=0}^{+\infty} \sum_{p=0}^{d-1} w_{p} x\left(\frac{l+\varepsilon_{p}}{n} e^{i \beta_{j}}\right) \frac{e^{i \beta_{j}}}{n}
\end{aligned}
$$

where $w_{p}$ and $\varepsilon_{p}$ are as in (1.5). We also need spline spaces on the contours $\Gamma_{j}$ and $\mathbb{R}^{+}$. Let $S_{n}^{\beta_{j}, \omega_{j}}$ be the smallest subspace of $L^{2}\left(\Gamma_{j}\right)$ which contains all functions

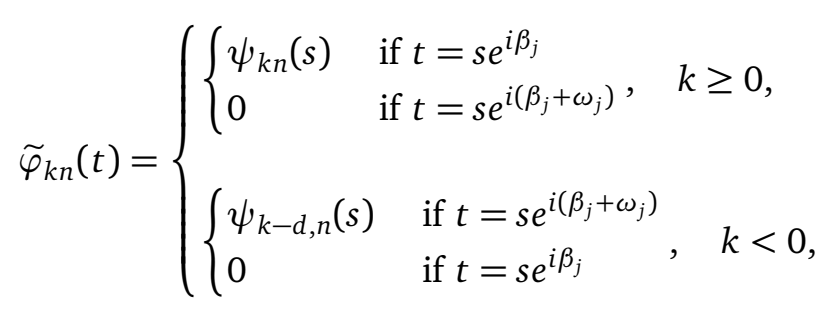


with $\psi_{k n}$ defined by (2.3).

The spline space $S_{n}\left(\mathbb{R}^{+}\right)=S_{n}^{d}\left(\mathbb{R}^{+}\right)$is constructed analogously but we let $\beta_{j}=0$ and use the splines $\widetilde{\varphi}_{k n}$ for $k \geq 0$ only. Moreover, let $\widetilde{P}_{n}$ and $\widehat{P}_{n}$ be the orthogonal projections from $L^{2}\left(\Gamma_{j}\right)$ onto $S_{n}^{\beta_{j}, \omega_{j}}$ and from $L^{2}\left(\mathbb{R}^{+}\right)$onto $S_{n}\left(\mathbb{R}^{+}\right)$, respectively. By $\mathbf{R}_{2}\left(\Gamma_{j}\right)$ we denote the set of functions on $\Gamma_{j}$ which are Riemann integrable on each finite part of $\Gamma_{j}$ and such that

$$
\begin{aligned}
\|f\|_{\mathbf{R}}=\|f\|_{L^{2}\left(\Gamma_{j}\right)} & +\left(\sum_{k=0}^{+\infty} \sup _{t \in e^{i\left(\beta_{j}+\omega_{j}\right)}[k, k+1]}|f(t)|^{2}\right)^{1 / 2} \\
& +\left(\sum_{k=0}^{+\infty} \sup _{t \in e^{i \beta_{j}[k, k+1]}}|f(t)|^{2}\right)^{1 / 2}<+\infty .
\end{aligned}
$$

Considering now the integral equation

$$
A_{\Gamma_{j}} x=f, \quad f \in \mathbf{R}_{2}\left(\Gamma_{j}\right)
$$

we replace $x$ by an element $x_{n} \in S_{n}^{\beta_{j}, \omega_{j}}$, apply quadratures (3.6) to the corresponding integrals and use the interpolation projections $\widetilde{Q}_{n}^{\varepsilon}: \mathbf{R}\left(\Gamma_{j}\right) \mapsto S_{n}^{\beta_{j}, \omega_{j}}$ defined by

$$
\begin{aligned}
& \widetilde{Q}_{n}^{\varepsilon} x\left(\tau_{l p}\right)=x\left(\tau_{l p}\right), \quad l \in \mathbb{Z}, p=0,1, \cdots, d-1 ; \\
& \tau_{l p}= \begin{cases}\frac{l+\varepsilon_{p}}{n} e^{i\left(\beta_{j}+\omega_{j}\right)} & \text { if } l<0, \\
\frac{l+\varepsilon_{p}}{n} e^{i \beta_{j}} & \text { if } l \geq 0 .\end{cases}
\end{aligned}
$$

As the result, we obtain the operator equations

$$
\widetilde{Q}_{n}^{\varepsilon} A_{\Gamma_{j}}^{(\varepsilon, n)} \widetilde{P}_{n} x_{n}=\widetilde{Q}_{n}^{\varepsilon} f, \quad x_{n} \in S_{n}^{\beta_{j}, \omega_{j}}, n \in \mathbb{N},
$$

equivalent to the following infinite systems of linear algebraic equations,

$$
\begin{aligned}
& x_{n}\left(\tau_{k r}\right)+\frac{1}{2 \pi i} \sum_{l=-\infty}^{-1} \sum_{p=0}^{d-1} w_{p} x_{n}\left(\tau_{l p}\right)\left(\frac{e^{i\left(\beta_{j}+\omega_{j}\right)}}{\tau_{l p}-\tau_{k r}}-\frac{e^{-i\left(\beta_{j}+\omega_{j}\right)}}{\overline{\tau_{l p}}-\overline{\tau_{k r}}}\right) \frac{1}{n} \\
& +\frac{1}{2 \pi i} \sum_{l=0}^{\infty} \sum_{p=0}^{d-1} w_{p} x_{n}\left(\tau_{l p}\right)\left(\frac{e^{i \beta_{j}}}{\tau_{l p}-\tau_{k r}}-\frac{e^{-i \beta_{j}}}{\overline{\tau_{l p}}-\overline{\tau_{k r}}}\right) \frac{1}{n} \\
= & f\left(\tau_{k r}\right), \quad k \in \mathbb{Z}, r=0,1, \cdots, d-1,
\end{aligned}
$$

where the expressions in the round brackets are set to be 0 if $k=l$ and $r=p$ simultaneously. Note that operators (3.7) can be written in a different form by using the integral representation (3.1) of the Mellin convolution operators $\mathscr{M}\left(\mathbf{n}_{\omega_{j}}\right)$. More precisely, let $\widehat{Q}_{n}^{\varepsilon}, n \in \mathbb{N}$ be the interpolation operators defined on the positive semi-axis. 
Lemma 3.1. If $\mathbf{k}_{\omega}$ is the function (3.5), then the sequence $\left(\widetilde{Q}_{n}^{\varepsilon} A_{\Gamma_{j}}^{(\varepsilon, n)} \widetilde{P}_{n}\right)_{n \in \mathbb{N}}$ is stable if and only if so is the sequence $\left(\widehat{A}_{\omega_{j}}^{\varepsilon, n} \operatorname{diag}\left(\widehat{P}_{n}, \widehat{P}_{n}\right)\right)$,

$$
\widehat{A}_{\omega_{j}}^{\varepsilon, n} \operatorname{diag}\left(\widehat{P}_{n}, \widehat{P}_{n}\right)=\left(\begin{array}{cc}
\widehat{P}_{n} & \widehat{Q}_{n}^{\varepsilon} \mathscr{M}^{(\varepsilon, n)}\left(\mathbf{k}_{\omega_{j}}\right) \widehat{P}_{n} \\
\widehat{Q}_{n}^{1-\varepsilon} \mathscr{M}^{(1-\varepsilon, n)}\left(\mathbf{k}_{\omega_{j}}\right) \widehat{P}_{n} & \widehat{P}_{n}
\end{array}\right) .
$$

Proof. Let $\eta: L^{2}\left(\Gamma_{j}\right) \mapsto L^{2}\left(\mathbb{R}^{+}\right)^{2}$ be the isomorphism defined in Section 3. It is easily seen that

$$
\eta \widetilde{Q}_{n}^{\varepsilon} \eta^{-1}=\operatorname{diag}\left(\widehat{Q}_{n}^{\varepsilon}, \widehat{Q}_{n}^{1-\varepsilon}\right), \quad \eta \widetilde{P}_{n} \eta^{-1}=\operatorname{diag}\left(\widehat{P}_{n}, \widehat{P}_{n}\right)
$$

and

$$
\eta A_{\Gamma_{j}}^{(\varepsilon, n)} \eta^{-1}=\eta \widetilde{P}_{n} \eta^{-1}+\eta V_{\Gamma_{j}}^{(\varepsilon, n)} \eta^{-1}=\left(\begin{array}{cc}
\widehat{P}_{n} & \mathscr{M}^{(\varepsilon, n)}\left(\mathbf{k}_{\omega_{j}}\right) \\
\mathscr{M}^{(1-\varepsilon, n)}\left(\mathbf{k}_{\omega_{j}}\right) & \widehat{P}_{n}
\end{array}\right) .
$$

The obvious identity $\eta\left(\widetilde{Q}_{n}^{\varepsilon} A_{\Gamma_{j}}^{(\varepsilon, n)} \widetilde{P}_{n}\right) \eta^{-1}=\left(\eta \widetilde{Q}_{n}^{\varepsilon} \eta^{-1}\right)\left(\eta A_{\Gamma_{j}}^{(\varepsilon, n)} \eta^{-1}\right)\left(\eta \widetilde{P}_{n} \eta^{-1}\right)$ completes the proof.

Let $l_{2}$ be the space of sequences $\left(\xi_{j}\right)_{j=0}^{\infty}$ of complex numbers $\xi_{j}, j=0,1, \cdots$ such that $\sum_{j=0}^{\infty}\left|\xi_{j}\right|^{2}<+\infty$. We now define the operators $E_{n}: l_{2} \mapsto S_{n}\left(\mathbb{R}^{+}\right)$and $E_{-n}: S_{n}\left(\mathbb{R}^{+}\right) \mapsto l_{2}$ by

$$
E_{n}\left(\left(\xi_{j}\right)_{j=0}^{\infty}\right)=\sum_{j=0}^{+\infty} \xi_{j} \widetilde{\varphi}_{j n}(t), \quad E_{-n}\left(\sum_{j=0}^{+\infty} \xi_{j} \widetilde{\varphi}_{j n}(t)\right)=\left(\xi_{j}\right)_{j=0}^{\infty} .
$$

The operators $E_{n}$ and $E_{-n}$ are bounded [6] and there is a constant $C$ such that

$$
\left\|E _ { n } \left|\left\||| E_{-n}\right\| \leq C \text { for all } n \in \mathbb{N} .\right.\right.
$$

This allows to rewrite the stability conditions for the sequence $\left(\widetilde{Q}_{n}^{\delta} A_{\Gamma_{j}}^{(\varepsilon, n)} \widetilde{P}_{n}\right)_{n \in \mathbb{N}}$ in a more convenient form. By $\widehat{E}_{n}$ and $\widehat{E}_{-n}$ we denote the diagonal operators

$$
\widehat{E}_{n}:=\operatorname{diag}\left(E_{n}, E_{n}\right), \quad \widehat{E}_{-n}:=\operatorname{diag}\left(E_{-n}, E_{-n}\right) .
$$

Corollary 3.2. The sequence $\left(\widetilde{Q}_{n}^{\varepsilon} A_{\Gamma_{j}}^{(\varepsilon, n)} \widetilde{P}_{n}\right)_{n \in \mathbb{N}}$ is stable if and only if the operator $B_{\omega_{j}, \varepsilon}=$ $\widehat{E}_{-1} \widehat{A}_{\omega_{j}}^{\varepsilon, 1} \operatorname{diag}\left(\widehat{P}_{1}, \widehat{P}_{1}\right) \widehat{E}_{1}$ is invertible.

Proof. Straightforward calculations show that the entries of the approximation operator $\widehat{E}_{-n} \widehat{A}_{\omega_{j}}^{\varepsilon, n} \operatorname{diag}\left(\widehat{P}_{n}, \widehat{P}_{n}\right) \widehat{E}_{n}$ do not depend on $n$. Indeed, consider for example, the sequence $\left(E_{-n} \widehat{Q}_{n}^{\varepsilon} \mathscr{M}^{(\varepsilon, n)}\left(\mathbf{k}_{\omega_{j}}\right) \widehat{P}_{n} E_{n}\right)$. If $x_{n} \in \operatorname{im} \widehat{P}_{n}$, then

$$
\begin{aligned}
\left(\mathscr{M}^{(\varepsilon, n)}\left(\mathbf{k}_{\omega_{j}}\right) x_{n}\right)(\sigma) & =\sum_{l=0}^{+\infty} \sum_{p=0}^{d-1} w_{p} \mathbf{k}_{\omega_{j}}\left(\frac{\sigma}{\frac{l+\varepsilon_{p}}{n}}\right) \frac{1}{\frac{l+\varepsilon_{p}}{n}} \frac{1}{n} x_{n}\left(\frac{l+\varepsilon_{p}}{n}\right) \\
& =\sum_{p=0}^{d-1} w_{p} \sum_{l=0}^{\infty} \mathbf{k}_{\omega_{j}}\left(\frac{\sigma}{\frac{l+\varepsilon_{p}}{n}}\right) \frac{1}{l+\varepsilon_{p}} x_{n}\left(\frac{l+\varepsilon_{p}}{n}\right),
\end{aligned}
$$


and applying the interpolation operators $\widetilde{Q}_{n}^{\varepsilon}$, we obtain

$$
\begin{aligned}
\left(\widetilde{Q}_{n}^{\varepsilon} \mathscr{M}^{(\varepsilon, n)}\left(\mathbf{k}_{\omega_{j}}\right) x_{n}\right)\left(\frac{k+\varepsilon_{r}}{n}\right) & =\sum_{p=0}^{d-1} w_{p} \sum_{l=0}^{\infty} \mathbf{k}_{\omega_{j}}\left(\frac{\frac{k+\varepsilon_{r}}{n}}{\frac{l+\varepsilon_{p}}{n}}\right) \frac{1}{l+\varepsilon_{p}} x_{n}\left(\frac{l+\varepsilon_{p}}{n}\right) \\
& =\sum_{p=0}^{d-1} w_{p} \sum_{l=0}^{\infty} \mathbf{k}_{\omega_{j}}\left(\frac{k+\varepsilon_{r}}{l+\varepsilon_{p}}\right) \frac{1}{l+\varepsilon_{p}} x_{n}\left(\frac{l+\varepsilon_{p}}{n}\right) .
\end{aligned}
$$

Thus the sequence in question is constant and is stable if and only if one its member, say $E_{-1} \widehat{A}_{\omega_{j}}^{\varepsilon, 1} \operatorname{diag}\left(\widehat{P}_{1}, \widehat{P}_{1}\right) E_{1}$, is invertible.

Theorem 3.2. If the operator $A: L^{2}(\Gamma) \mapsto L^{2}(\Gamma)$ is invertible, then the Nyström method for $A$ is stable if and only if all the operators $B_{\omega_{j}, \varepsilon}, j=0,1, \cdots, q-1$ are invertible.

Proof. Let $\mathscr{C}$ denote the smallest closed $C^{*}$-algebra containing the sequences $\left(P_{n} S_{\Gamma} P_{n}\right)$, $\left(P_{n} M P_{n}\right)$ and $\left(P_{n} f P_{n}\right)$, where $f \in C(\Gamma)$, and let $\mathscr{J}$ be the ideal defined in Theorem 3.1. Then $\left(A^{(\varepsilon, n)} P_{n}\right) \in \mathscr{C}$ and $\mathscr{C} / \mathscr{J}$ is a $C^{*}$-subalgebra of $\mathscr{A} / \mathscr{J}$. Therefore, the coset $\left(A^{(\varepsilon, n)} P_{n}\right)+\mathscr{J}$ is invertible in $\mathscr{A} / \mathscr{J}$ if and only if it is invertible in $\mathscr{C} / \mathscr{J}$. However, the algebra $\mathscr{C} / \mathscr{J}$ has a nice centre and the invertibility of the $\operatorname{coset}\left(A^{(\varepsilon, n)} P_{n}\right)+\mathscr{J}$ in $\mathscr{C} / \mathscr{J}$ can be established by the Allan's local principle - cf. Ref. [1] and [14, Theorem 1.9.5]. Following the proof of Theorem 3.4 in Ref. [11] we show that for any $\tau=\tau_{j} \in \mathfrak{M}_{\Gamma}$ this coset is invertible if and only if the corresponding operator $B_{\omega_{j}, \varepsilon}$ is invertible. On the other hand, it was already mentioned that for $\tau \notin \mathfrak{M}_{\Gamma}$, the corresponding coset is always invertible, and application of Theorem 3.1 completes the proof.

\section{The Invertibility of Local Operators}

Theorem 3.2 connects the stability of the Nyström method with the invertibility of the operators $B_{\omega_{j}, \varepsilon}, j=0,1, \cdots, q-1$. A more detailed study of these operators shows that they belong to a Toeplitz algebra with no efficient invertibility conditions available. Similar problems arise in approximation methods for the Sherman-Lauricella and Muskhelishvili equations. A numerical approach to the invertibility of related operators has been proposed in $[9,10]$, with the invertibility connected to the stability of the approximation method for operators on model curves with all corners of the same magnitude $\omega$. Looking at the condition numbers of the corresponding method, one can decide which opening angles $\omega$ are "critical" - i.e. which angles cause instability.

Theorem 4.1. Let $\mathscr{L}=\mathscr{L}(\omega)$ be any curve $\mathscr{L}_{1}(\omega)$ or $\mathscr{L}_{2}(\omega), \omega \in(0,2 \pi)$ defined in Section 3 and $A_{\mathscr{L}(\omega)}$ be the operator (2.6) considered on the contour $\mathscr{L}(\omega)$. The operator $B_{\omega, \varepsilon}$ is invertible if and only if the Nyström method $\left(Q_{n}^{\varepsilon} A_{\mathscr{L}(\omega)}^{(\varepsilon, n)} P_{n}\right)$ is stable.

This statement is a reformulation of Theorem 3.2 with respect to the chosen operator and special contours. Of course, we take into account the invertibility of $A_{\mathscr{L}(\omega)}$ : 

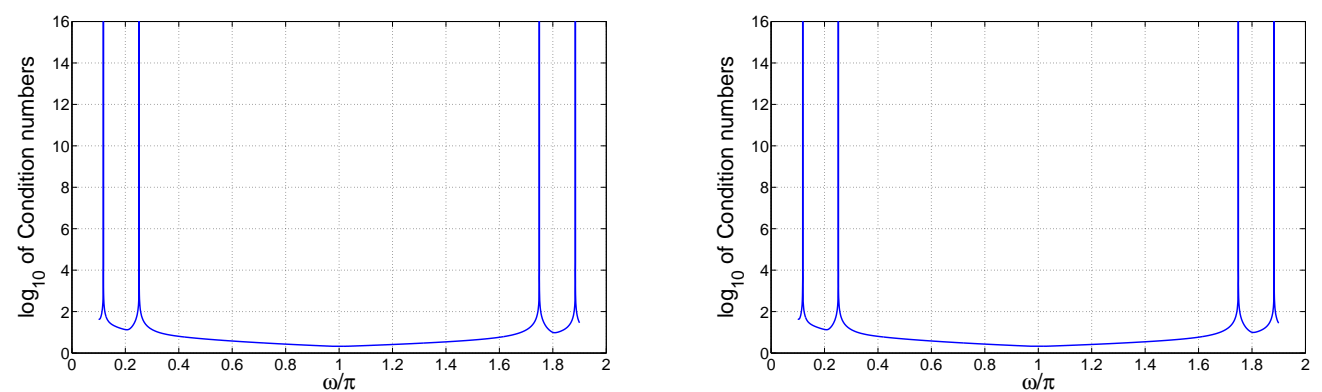

Figure 4: Condition numbers vs. opening angles in case $n=128, d=16$. Left: one corner contour. Right: two corner contour.

$L^{2}(\mathscr{L}(\omega)) \rightarrow L^{2}(\mathscr{L}(\omega))-$ cf. Ref. [27]. Let us emphasise that $\mathscr{L}_{1}$ and $\mathscr{L}_{2}$ have distinct shapes and a different number of corners. However, if the magnitude of the corners is the same, the corresponding numerical experiments shall produce the same results. In what follows, we vary the parameter $\omega$ in the interval $(0.1 \pi, 1.9 \pi)$ and obtain two families of contours with one and two corners. In order to find critical angles, we divide the interval $[0.1 \pi, 1.9 \pi]$ by the points $\omega_{k}=\pi(0.1+0.001 k)$, and for each point $\omega_{k}$ we compute the condition number of the Nyström method for $n=128$ and the Gauss-Legendre quadrature with $d=16$ - viz. there are 128 panels on the curves and each panel has 16 mesh points. Computing the corresponding condition numbers at the points $\omega_{k}$, we first detect "suspicious" points, in the neighbourhoods of which condition numbers grow rapidly. Thereafter, the initial mesh is refined in neighborhoods of such points and condition numbers are recalculated. The procedure is repeated until the related condition numbers reach $10^{16}$. The outcome of these computations is presented in Fig. 4. Thus using both contours we found that the corresponding graphs have four peaks in the interval $(0.1,1.9)-$ viz.

The case of one corner geometry - curve $\mathscr{L}_{1}$.

$$
0.11781222 \pi, \quad 0.25164815 \pi, \quad 1.74949877 \pi, \quad 1.88430019 \pi \text {. }
$$

The case of two corner geometry - curve $\mathscr{L}_{2}$.

$$
0.11780844 \pi, \quad 0.25164706 \pi, \quad 1.74840993 \pi, \quad 1.88390254 \pi \text {. }
$$

Let us emphasise that for both curves $\mathscr{L}_{1}$ and $\mathscr{L}_{2}$, the results coincide up to three significant numbers. The peaks of the graphs are connected with four possible critical angles in the interval $(0.1 \pi, 1.9 \pi)$.

The numerical experiments have been conducted in MATLAB environment (version 7.9.0) and executed on an Acer Veriton M680 workstation equipped with a Intel Core i7 vPro 870 processor and $8 \mathrm{~GB}$ of RAM.

\section{Conclusion}

We established necessary and sufficient conditions for stability of the Nyström method for double layer potential equations on simple piecewise smooth contours. In particular, 
we found four opening angles in the interval $[0.1 \pi, 1.9 \pi]$ which cause the instability. Thus if $\Gamma$ has a corner of such magnitude then, regardless of the shape of the curve, the Nyström method is not stable even if the operator $A$ of (1.2) is invertible. In numerical experiments we used curves with different number of corners of the same magnitude and all of them produced the same critical angles, consistent with theoretical analysis.

\section{References}

[1] G.R. Allan, Ideals of vector-valued functions, Proc. London Math. Soc. 18, 193-216 (1968).

[2] K.E. Atkinson, The Numerical Solution of Integral Equations of the Second Kind, Cambridge University Press (1997).

[3] M.D. Bonis and C. Laurita, A modified Nyström method for integral equations with Mellin type kernels, J. Comput. Appl. Math. 296, 512 - 527 (2016).

[4] J. Bremer, On the Nyström discretization of integral equations on planar curves with corners, Appl. Comput. Harmon. Anal. 32, 45-64 (2012).

[5] J. Bremer and V. Rokhlin, Efficient discretization of Laplace boundary integral equations on polygonal domains, J. Comput. Phys. 229, 2507-2525 (2010).

[6] C. de Boor, A Practical Guide to Splines, Springer Verlag (1978).

[7] G.A. Chandler and I.G. Graham, Higher order methods for linear functionals of solutions of second kind integral equations, SIAM J. Num. Anal. 25, 1118-1137 (1988).

[8] M. Costabel, Boundary integral operators on Lipschitz domains: elementary results, SIAM J. Math. Anal. 19, 613-626 (1988).

[9] V.D. Didenko and J. Helsing, Features of the Nyström method for the Sherman-Lauricella equation on piecewise smooth contours, East Asian J. Appl. Math. 1, 403-414 (2011).

[10] V.D. Didenko and J. Helsing, Stability of the Nyström method for the Sherman-Lauricella equation, SIAM J. Numer. Anal. 49, 1127-1148 (2011).

[11] V.D. Didenko and J. Helsing, On the stability of the Nyström method for the Muskhelishvili equation on contours with corners, SIAM J. Numer. Anal. 51, 1757-1776 (2013).

[12] V.D. Didenko, S. Roch and B. Silbermann, Approximation methods for singular integral equations with conjugation on curves with corners, SIAM J. Numer. Anal. 32, 1910-1939 (1995).

[13] V.D. Didenko and B. Silbermann, An approximate method on non-equidistant partitions for double layer potential equation, Appl. Numer. Math. 26, 41-48 (1998).

[14] V.D. Didenko and B. Silbermann, Approximation of Additive Convolution-Like Operators. Real $C^{*}$-Algebra Approach, Frontiers in Mathematics, Birkhäuser Verlag (2008).

[15] V.D. Didenko, T. Tao and A.M. Vu, Spline Galerkin methods for the Sherman-Lauricella equation on contours with corners, SIAM J. Numer. Anal. 53, 2752-2770 (2015).

[16] V.D. Didenko and E. Venturino, Approximation method for the Muskhelishvili equation on smooth curves, Math. Comp. 76, 1317-1339 (2007).

[17] V.D. Didenko and A.M. Vu, Spline Galerkin methods for the double layer potential equations on contours with corners, in Recent Trends in Operator Theory and Partial Differential Equations. The Roland Duduchava Anniversary Volume, Oper. Theory Adv. Appl. 258, pp. 129-144, Birkhäuser Verlag (2017).

[18] L. Fermo and C. Laurita, On the numerical solution of a boundary integral equation for the exterior Neumann problem on domains with corners, Appl. Numer. Math. 94, 179-200 (2015).

[19] R. Hagen, S. Roch and B. Silbermann, Spectral theory of approximation methods for convolution equations, Operator Theory: Advances and Applications 74, Birkhäuser Verlag (1995). 
[20] J. Helsing and A. Holst, Variants of an explicit kernel-split panel-based Nyström discretization scheme for Helmholtz boundary value problems, Adv. Comput. Math. 41, 691-708 (2015).

[21] R. Kress, A Nyström method for boundary integral equations in domains with corners, Numer. Math. 58, 145-161 (1990).

[22] R. Kress, Linear Integral Equations, Vol. 82 of Applied Mathematical Sciences, Springer Verlag (2014).

[23] N.I. Muskhelishvili, Singular Integral Equations, Nauka (1968).

[24] S. Prössdorf and B. Silbermann, Numerical Analysis for Integral and Related Operator Equations, Birkhäuser Verlag (1991).

[25] S. Roch, P.A. Santos and B. Silbermann, Non-commutative Gelfand Theories. A Tool-Kit for Operator Theorists and Numerical Analysts, Universitext, Springer-Verlag (2011).

[26] L.L. Schumaker, Spline Functions: Basic Theory, Krieger Publishing (1993).

[27] G. Verchota, Layer potentials and regularity for the Dirichlet problem for Laplace's equation in Lipschitz domains, J. Funct. Anal. 59, 572-611 (1984). 